\title{
Precision medicine in pediatric oncology
}

\author{
Suzanne J. Forrest ${ }^{\mathrm{a}}$, Birgit Geoerger ${ }^{\mathrm{b}, \mathrm{c}}$, and Katherine A. Janeway ${ }^{\mathrm{a}}$
}

\begin{abstract}
Purpose of review
The current review describes recent advances and unique challenges in precision medicine for pediatric cancers and highlights clinical trials assessing the clinical impact of targeted therapy matched to molecular alterations identified by tumor profiling.

\section{Recent findings}

Multiple prospective clinical sequencing studies in pediatric oncology have been reported in the last 2 years. These studies demonstrated feasibility of sequencing in the clinic and revealed a rate of actionable variants that justifies the development of precision trials for childhood cancer. A number of precision medicine trials are recently completed, underway or in development and these will be reviewed herein, with a focus on highlighting aspects of precision medicine trial design relevant to pediatric oncology.
\end{abstract}

\section{Summary}

The primary results of the first round of pediatric precision oncology clinical trials will provide us with a greater understanding of the clinical impact of linking tumor profiling to selection of targeted therapies. The aggregation of sequencing and clinical data from these trials and the results of biologic investigations linked to these trials will drive further discoveries and broaden opportunities for precision medicine for children with cancer.

\section{Keywords}

basket trial, molecular profiling, pediatric cancer, precision medicine, targeted therapy

\section{INTRODUCTION}

In the last 50 years, there has been significant improvement in outcomes for children with cancer, driven primarily by risk stratification and intensification of cytotoxic chemotherapy coupled with multimodality treatment approaches [1]. Yet there is still a need to improve outcomes for all children with cancer, with attention focused on increasing survival in those with a poor prognosis and decreasing serious late side effects of multimodality therapy.

The current review focuses on the current approach in pediatric oncology to assessment of the cancer genome for the purpose of incorporating targeted therapies in clinical trials and ultimately standard of care treatment. The ultimate goal of precision medicine is the same in pediatric oncology as it is in other specialties: to cure more patients and minimize both short and long-term side effects associated current treatments [2]. As precision oncology is a new field, we have defined commonly used terms utilized throughout this review in Table 1.

Over the past decade, next generation sequencing was employed as one of the initials steps in defining the genomic features of the most common pediatric cancer diagnoses. The completed discovery sequencing studies were reviewed at the National Cancer Institute (NCI) Childhood Cancer Genomics Gaps and Opportunities Workshop in February 2015 [3]. An updated list of discovery sequencing studies in pediatric malignancies is in Supplementary Table 1 , http://links.lww.com/MOP/A29. Despite ongoing progress in large-scale discovery sequencing projects in pediatric cancers, for the majority of pediatric cancer diagnoses, only a small number of tumor-normal pairs have been subjected to comprehensive sequencing [4-8]. As a result, for most diagnoses, these discovery sequencing studies are underpowered to rule out the presence of recurrent mutations occurring

aPediatrics, Dana-Farber/Boston Children's Cancer and Blood Disorders Center, Boston, Massachusetts, USA, ${ }^{b}$ Department of Pediatric and Adolescent Oncology, Gustave Roussy and ${ }^{\circ}$ Gustave Roussy, Department of Pediatric and Adolescent Oncology, Université Paris-Saclay, Univ Paris Sud, CNRS UMR8203, Villejuif, France

Correspondence to Katherine A. Janeway, MD, MSc, Dana-Farber/ Boston Children's Cancer and Blood Disorders Center, 450 Brookline Ave, Dana 3, Boston, MA 02215, USA. Tel: +1 617632 4994; e-mail: Katherine_janeway@dfci.harvard.edu

Curr Opin Pediatr 2018, 30:17-24

DOI:10.1097/MOP.0000000000000570

This is an open access article distributed under the terms of the Creative Commons Attribution-Non Commercial-No Derivatives License 4.0 (CCBY-NC-ND), where it is permissible to download and share the work provided it is properly cited. The work cannot be changed in any way or used commercially without permission from the journal. 


\section{KEY POINTS}

- Clinical sequencing studies completed in the last 2 years have set the stage for precision clinical trials designed to assess the impact of molecularly targeted therapies in pediatric oncology.

- Precision oncology trials of varying design are currently underway in pediatric oncology, including major initiatives in the United States and Europe resulting in basket trials in relapsed or refractory pediatric cancers.

- Aggregation of sequencing, clinical and correlative biology data from targeted therapy trials will lead to further discoveries and increased opportunities for precision medicine in children with cancer.

with an incidence as high as $5-10 \%$ of cases [9]. In addition, the genomics of many rare pediatric cancer types have not been adequately studied and few specimens from recurrent disease have been sequenced.

Completed discovery sequencing studies have revealed important aspects of the pediatric cancer genome. Compared with adult cancers, pediatric cancers harbor far fewer amino acid changing mutations per megabase of DNA [10]. Segmental chromosomal changes, fusions and mutations in genes impacting the epigenome are important genomic mechanisms of malignant transformation in childhood cancers. In addition to these observations, discovery sequencing studies have furthered molecular classification of some diseases including identification of high-risk genomic features that are associated with prognosis and guide treatment stratification in several common pediatric malignancies such as leukemia and medulloblastoma.

However, discovery sequencing studies are not designed to assess the feasibility of the routine performance of genomic profiling in the clinic, nor do they evaluate the ability to analyze and interpret individual patient-level sequencing results that will inform approaches to patient treatment and clinical trials selection. Clinical sequencing studies, in which sequencing results are returned usually after the testing is performed in a clinical laboratory, are required to address these questions. In the last 2 years, seven pediatric oncology clinical sequencing studies have been published (Table 2) [11-17]. The first of these were comprehensively reviewed by Mody et al. [18"], and so we will only briefly touch on the conclusions [19"',20,21",22,23].

The completed clinical sequencing studies, which enrolled children and adolescents with relapsed, refractory or high-risk solid tumors, demonstrated the feasibility of clinical sequencing at a single site or across multiple sites using a central laboratory. The technical success rate was high even when specimens collected for clinical purposes were used for sequencing. In addition, practices for returning results to treating providers and patient families (often including germline testing) were established and patient and provider perceptions of tumor profiling and germline sequencing are beginning to be assessed [24]. The diverse sequencing platforms used in each study resulted in similar rates (30-60\%) of potentially actionable variants. When assessed, relatively few patients in these initial studies (3-18\% of the study populations) received targeted therapy matched to an identified actionable variant. This is not surprising as the study design and limited duration of clinical follow-up preclude the evaluation of the impact of receipt of matched targeted treatments on outcomes. Nevertheless, these initial clinical sequencing studies set the stage for subsequent precision clinical trials that are prospectively designed to assess the impact of molecularly targeted therapies in pediatric oncology [19"'].

\section{PRECISION MEDICINE TRIALS}

The design of precision oncology trials must be responsive to rapid evolutions in genomic technologies, our understanding of the landscape of the cancer genome, and the availability of targeted therapeutics.

Table 1. Glossary of precision oncology terms

\footnotetext{
Somatic gene variant: Single nucleotide variant (SNV), copy number variant (CNV) or rearrangement identified in tumor. Of note, when tumor only sequencing is performed it can be difficult to know whether an identified gene variant, in particular an SNV, is somatic or germline

Actionable variant: Gene variant determined to be likely to alter protein function and with evidence linking that variant to a response to targeted therapy

Targeted therapy: Drug with known and specific mechanism of action

Evidence: Results of pre-existing research used to link genomic variants to clinical significance such as potential for response to targeted therapy

Precision medicine trial: Clinical trial in which patient selection/eligibility for receipt of targeted therapy is determined by presence or absence of biomarker

Biomarker: Gene variant or test measuring the presence of the gene variant that is expected to predict response to targeted therapy
} 
Table 2. Published clinical sequencing studies in pediatric oncology

\begin{tabular}{|c|c|c|c|c|c|c|c|}
\hline Study & Institution & Tumor types & Patient population & $\begin{array}{l}\text { No. of patients } \\
\text { analyzed }\end{array}$ & $\begin{array}{l}\text { \% Actionable } \\
\text { alterations }\end{array}$ & $\begin{array}{c}\text { Year } \\
\text { published }\end{array}$ & PMID \\
\hline $\begin{array}{l}\text { Peds- } \\
\text { MiOncoSeq }\end{array}$ & University of Michigan & $\begin{array}{l}\text { Solid (including CNS) } \\
\text { and hematologic } \\
\text { malignancies }\end{array}$ & $\begin{array}{l}\text { Relapsed/refractory, } \\
\text { high-risk newly } \\
\text { diagnosed }\end{array}$ & 91 & 46 & 2015 & 26325560 \\
\hline iCat & $\begin{array}{l}\text { Dana-Farber Cancer } \\
\text { Institute and others }\end{array}$ & $\begin{array}{l}\text { Extracranial solid } \\
\text { tumors }\end{array}$ & $\begin{array}{l}\text { Relapsed/refractory, } \\
\text { high-risk newly } \\
\text { diagnosed }\end{array}$ & 89 & 34 & 2016 & 26822149 \\
\hline BASIC3 & $\begin{array}{l}\text { Baylor College of } \\
\text { Medicine }\end{array}$ & Solid (including CNS) & Newly diagnosed & 150 & 39 & 2016 & 26822237 \\
\hline INFORM & $\begin{array}{l}\text { German Cancer } \\
\text { Research Center and } \\
\text { others }\end{array}$ & $\begin{array}{l}\text { Solid (including CNS) } \\
\text { and hematologic } \\
\text { malignancies }\end{array}$ & Relapsed/refractory & 52 & 50 & 2016 & 27479119 \\
\hline MBB Program & Institut Curie, France & Solid (including CNS) & $\begin{array}{l}\text { Relapsed/refractory, } \\
\text { high-risk newly } \\
\text { diagnosed }\end{array}$ & 58 & 40 & 2016 & 27896933 \\
\hline PIPseq & $\begin{array}{l}\text { Columbia University } \\
\text { Medical Center }\end{array}$ & $\begin{array}{l}\text { Solid (including CNS) } \\
\text { and hematologic } \\
\text { malignancies }\end{array}$ & $\begin{array}{l}\text { Relapsed/refractory, } \\
\text { high-risk newly } \\
\text { diagnosed }\end{array}$ & 101 & 38 & 2016 & 28007021 \\
\hline MOSCATO-01 & Gustave Roussy, France & Solid (including CNS) & Relapsed/refractory & 69 & 61 & 2017 & 28733441 \\
\hline
\end{tabular}

CNS, central nervous system, INFORM, individualized therapy for relapsed malignancies.

The regulatory environment is another factor that may be associated with unanticipated changes. As a result of these and other factors, precision oncology trial designs, mostly developed in the context of adult cancers, are still constantly changing. Despite this, we have attempted to classify the pediatric precision trials discussed below into four groups (Table 3 ) based on patient population (eligibility) and design. We provide examples of some of the precision medicine trials in pediatric oncology as a way of highlighting the key aspects of precision medicine trial design relevant to pediatric oncology $[25,26]$.

\section{Basket trials in relapsed/refractory cancers across multiple histologies}

Precision trials with the largest scope are referred to as 'basket trials'. These trials span cancer diagnoses and the results of sequencing are used to identify actionable variants linked to eligibility for clinical trial arms of targeted therapeutics. A basket trial is the ideal design when genomic variants that may predict response to a targeted therapy of interest occur at either a low or unknown frequency across diseases. Our limited understanding of the frequency of genomic variants and their oncogenic

Table 3. Examples of precision trials in pediatric oncology

\begin{tabular}{|c|c|c|c|}
\hline Trial type & Examples of precision trials & Sponsor & ClinicalTrials ID \\
\hline $\begin{array}{l}\text { Basket in relapsed/refractory cancers } \\
\text { across multiple diagnoses }\end{array}$ & $\begin{array}{l}\mathrm{NCl}-\mathrm{COG} \text { Pediatric MATCH } \\
\text { AcSé-ESMART }\end{array}$ & $\begin{array}{l}\mathrm{COG} / \mathrm{NCl} \\
\text { Gustave Roussy }\end{array}$ & $\begin{array}{l}\text { NCT03155620 } \\
\text { NCT02813135 }\end{array}$ \\
\hline $\begin{array}{l}\text { Disease-specific umbrella in patients } \\
\text { with progressive disease }\end{array}$ & $\begin{array}{l}\text { Ruxolitinib or Dasatinib with Chemotherapy in Ph-Like ALL } \\
\text { NEPENTHE (Neuroblastoma) }\end{array}$ & $\begin{array}{l}\text { MD Anderson } \\
\text { CHOP }\end{array}$ & $\begin{array}{l}\text { NCT02420717 } \\
\text { NCT02780128 }\end{array}$ \\
\hline $\begin{array}{l}\text { Single-agent targeted therapy in } \\
\text { advanced cancers }\end{array}$ & $\begin{array}{l}\text { Larotrectinib in NTRK Fusion Positive Tumors } \\
\text { EZH2 Inhibitor Tazemetostat in INI-1 Negative tumors } \\
\text { Crizotinib for Tumors with an ALK, MET or ROS1 alteration } \\
\text { LDK378 (Ceritinib) in ALK-activated Pediatric Tumors } \\
\text { Dabrafenib with Trametinib for BRAF V600 Positive Tumors } \\
\text { Afatinib in Pediatric Tumors with ErbB Pathway Deregulation }\end{array}$ & $\begin{array}{l}\text { LOXO Oncology } \\
\text { Epizyme } \\
\text { UNICANCER } \\
\text { Novartis } \\
\text { Novartis } \\
\text { Boehringer } \\
\quad \text { Ingelheim }\end{array}$ & $\begin{array}{l}\text { NCT02637687 } \\
\text { NCT02601937 } \\
\text { NCTO2034981 } \\
\text { NCT01742286 } \\
\text { NCT02684058 } \\
\text { NCT02372006 }\end{array}$ \\
\hline $\begin{array}{l}\text { Disease-specific trials in newly } \\
\text { diagnosed patients }\end{array}$ & $\begin{array}{l}\text { Total Therapy XVII JAK/STAT Mutations in ALL and Lymphoma } \\
\text { Addition of Dasatinib for ALL with TKI-targetable Fusions } \\
\text { Combination Therapy Plus Dasatinib for Ph-Like B-ALL } \\
\text { Clinical and Molecular Risk-Directed Therapy (Medulloblastoma) } \\
\text { BIOMEDE (DIPG) }\end{array}$ & $\begin{array}{l}\text { St. Jude } \\
\text { DFCl } \\
\text { COG/NCl } \\
\text { St. Jude } \\
\text { Gustave Roussy }\end{array}$ & $\begin{array}{l}\text { NCTO3 } 117751 \\
\text { NCT03020030 } \\
\text { NCT02883049 } \\
\text { NCTO1878617 } \\
\text { NCT02233049 }\end{array}$ \\
\hline
\end{tabular}

ALL, acute lymphoblastic leukemia; AcSé-ESMART, Secured Access Program of the French National Cancer Institute (INCa); COG, Children's Oncology Group; ESMART, European Proof-of-Concept Therapeutic Stratification Trial of Molecular Anomalies in Relapsed or Refractory Tumors in Children; MATCH, Molecular Analysis for Therapy Choice; NCl, National Cancer Institute; NEPENTHE, Next Generation Personalized Neuroblastoma Therapy; Ph-like, Philadelphia chromosome-like. 
Table 4. Differences in Children's Oncology Group-National Cancer Institute Pediatric Molecular Analysis for Therapy Choice trial and AcSé-European Proof-of-Concept Therapeutic Stratification Trial of Molecular Anomalies in Relapsed or Refractory Tumors in Children trial designs

\begin{tabular}{lll} 
& Pediatric-MATCH & AcSé-ESMART \\
\hline Sequencing platform & One uniform targeted panel & Multiple panels from different sequencing studies \\
Matching method & Automated matching & Tumor board discussion \\
Treatments & Single-agent & Combination with chemotherapy \\
Targets (drug) & TSC1, TSC2, PI3K/mTOR (LY3023414) & mTORC1/TORC2 (Arm 1 AZD2014) \\
& Mismatch repair (Olaparib) & mTORC1/TORC2 (Arm 2 AZD2014+ Topotecan + Temozolomide) \\
& NTRK1/2/3 (Larotrectinib) & Mismatch repair defect in WEE 1 (AZD1775 + Carboplatin) \\
& EZH2, SMARCB1, SMARCA4 (Tazemetostat) & Mismatch repair defect in PARP (Olaparib + Irinotecan) \\
& MAPK pathway (Selumetinib) & CDK4/6 (Arm 1 Ribociclib and Topotecan + Temozolomide) \\
& ALK, ROS1 (Ensartinib) & CDK4/6 (Arm 2 Ribociclib and Everolimus) \\
& BFAF V600 (Vemurafenib) & PD1 (Nivolumab + cyclophosphamide \pm radiation)
\end{tabular}

AcSé-ESMART, Secured Access Program of the French National Cancer Institute (INCa); ESMART, European Proof-of-Concept Therapeutic Stratification Trial of Molecular Anomalies in Relapsed or Refractory Tumors in Children; mTOR, mammalian target of rapamycin, PARP, poly ADP ribose polymerase.

potential in many pediatric cancers means basket trials play an important role in the development of targeted therapies in pediatric oncology.

Now underway in the United States and Europe are major basket trial initiatives for children and adolescents with relapsed or refractory pediatric cancers. In the United States, the Children's Oncology Group (COG)-NCI Pediatric MATCH (Molecular Analysis for Therapy Choice) trial, opened for patient enrollment in July 2017 [27"']. The Pediatric MATCH was developed following initiation of a counterpart trial for adult cancer patients, the NCI-MATCH trial [28-30]. Children and adolescents aged 1-21 years with recurrent or refractory solid tumors, non-Hodgkin lymphomas or histiocytoses are eligible for enrollment. A sample of the patients' relapsed tumor (except for diffuse intrinsic pontine glioma in which a diagnostic biopsy is allowed) is submitted for sequencing utilizing an assay developed specifically for the MATCH trial. The sequencing platform utilizes amplicon technology for mutation detection and dual primer multiplexed RNA-based PCR for fusion detection. Sequencing data is analyzed to determine whether an actionable mutation of interest is present. Actionable mutations of interest are predetermined for each trial arm. There are currently seven treatment arms (Table 4), with additional arms expected to open in the future.

Treatment arms are selected for inclusion in the pediatric COG-NCI Pediatric MATCH if there is already existing evidence from a clinical trial linking gene variants to response to the targeted therapy. For targeted therapy arms in which clinical evidence exists but is more limited, such as case reports, there also needs to be laboratory preclinical investigation providing evidence that the gene variants are credible biomarkers of response to the targeted therapy. Of note, the clinical evidence utilized does not need to be from the same cancer type as the hypothesis being tested in a basket trial is that the biomarker will predict response to targeted therapy regardless of cancer type. In the COG-NCI Pediatric MATCH trial, at least 20 patients will be enrolled in each treatment arm and the primary outcome measure for each trial arm is objective response rate (RR).

The European Proof-of-Concept Therapeutic Stratification Trial of Molecular Anomalies in Relapsed or Refractory Tumors in Children [AcSéESMART (Secured Access Program of the French National Cancer Institute (INCa)] opened for enrollment in August 2016. The ESMART trial is part of the Innovative Therapies for Children with Cancer Precision Cancer Medicine Program, a European academic consortium [31"',32,33]. Children and adolescents aged $0-18$ years with relapsed or refractory solid tumors and leukemias are eligible. Patients must have had advanced molecular profiling of their recurrent or refractory tumor performed in one of the ongoing clinical sequencing studies in Europe such as A multicentric, prospective proof-of-concept study MoleculAr Profiling for Pediatric and Young Adult Cancer Treatment Stratification or INdividualized therapy FOr Relapsed Malignancies. Most of these European clinical sequencing studies subject samples to multiple sequencing platforms including whole exome sequencing, RNA sequencing, and whole genome sequencing. Sequencing data are reviewed and examined at a multidisciplinary molecular tumor board to determine whether an actionable variant is present and whether the actionable variant is a match for one of the ESMART trial arms or other targeted agent trials. Currently, ESMART has seven treatment arms for five genomic 
targets/pathways (Table 4). Each of the treatment arms are run as an independent clinical trial, with a phase 1 dose escalation phase and a phase 2 expansion phase. Accordingly, each arm will enroll six to 38 patients. Unlike the Pediatric MATCH trial, many of the treatment arms include a targeted therapy agent in combination with another targeted agent or conventional cytotoxic chemotherapy. In addition to clearly defined biomarkers, the trial intends to explore through an enrichment strategy the role of nonwell defined molecular alterations in regard to their sensitivity to the targeted treatment. The ESMART trial enrolled 31 patients in the first 6 months [34].

The juxtaposition of the Pediatric MATCH and ESMART trials (Table 4) reveals variability in approaches to basket trial design. Each design has unique strengths and weaknesses. Thus, efforts to compare results, particularly if primary clinical and sequencing data are shared across continents, will lead to additional insights useful to inform design of future basket trials in pediatric oncology.

\section{Disease-specific umbrella trials in relapsed disease}

The term umbrella trial has been used to mean a basket trial limited to a single diagnosis. An umbrella design may be preferable when previous discovery sequencing has led to a relatively good understanding of the potentially actionable mutations seen in a particular diagnosis and the frequency of such variants is high enough that a study in that disease alone is feasible. Umbrella trials in relapsed disease are currently underway in neuroblastoma, and leukemia, which is not surprising as these are the pediatric cancers for which there has been a relatively large number of tumor-normal pairs subjected to discovery sequencing.

Discovery sequencing studies in acute lymphoblastic leukemia (ALL) led to the identification and characterization of Philadelphia chromosome-like (Ph-like) ALL $[4,35]$, a molecular subtype occurring in approximately $15 \%$ of children, adolescents and young adults and associated with a significantly poorer outcome $[36,37]$. Ph-like ALL is characterized by presence of a kinase-activated gene expression profile similar to $\mathrm{BCR}-\mathrm{ABL}$ translocation positive leukemia but without the BCR-ABL fusion. Ph-like ALLs have gene variants that activate kinase or cytokine signaling including rearrangements involving ABL1/2, CSF1R, PDGFRB and rearrangements and mutations in CRLF2, JAK2 and EPOR [4]. Preclinical studies and case reports suggested sensitivity to targeted treatments with JAK inhibitors or tyrosine kinase inhibitors $[37,38]$.
A single institution trial enrolling patients 10 years and older with relapsed or refractory ALL with Ph-like genetic lesion(s) or evidence of CRLF2 positivity by flow cytometry is combining reinduction chemotherapy with either ruxolitinib, a Jak inhibitor or dasatinib, an Abl/Src kinase inhibitor based on the gene variants identified [39]. It is interesting to note that this biomarker - driven phase 2 study is, from study initiation, enrolling patients 10 and above. Such an approach, in which eligibility criteria are expanded to allow younger patients to enroll in earlier phase trials, has been supported in recent statements from the US Food and Drug Administration (FDA) and from a multistakeholder task force including the American Society of Clinical Oncology $[40,41]$.

ALK is mutated in approximately $10 \%$ of neuroblastomas [7]. Sequencing of paired diagnostic and relapsed samples has revealed a higher rate of ALK mutations at relapse of approximately $25 \%$ [42-45]. In-vitro screening of eight targeted agents in 17 well characterized human neuroblastomaderived cell lines revealed sensitivity of ALK mutated lines to the combination of CDK4 inhibitor ribociclib and ALK inhibitor ceritinib [46]. Based on this and other preclinical evidence, the Next Generation Personalized Neuroblastoma Therapy trial was designed. Patients are eligible if they have relapsed or refractory neuroblastoma and are age 1-21 years. Biopsy is performed for tumor assessment with deep sequencing to identify mutations in the ALK or RAS-MAPK pathways. Patients whose tumors have an ALK pathway mutation receive a combination of ceritinib and ribociclib, and those with a RAS-MAPK alteration receive trametinib. Patients without an ALK or RAS/MAPK pathway alteration and with wild-type TP53, are enrolled onto the third treatment arm and receive HDM201, an oral HDM2 inhibitor.

\section{Single-agent targeted therapy in advanced cancers}

Early phase trials of a single targeted agent when eligibility criteria include a genomic biomarker are contributing to our understanding of genomics and activity of targeted agents in pediatric cancers. These trials tend to be industry sponsored and often utilize drugs primarily developed for gene variants present in the more common adult cancers. Previously, this type of pediatric early phase trial was often conducted several years after the therapy was proven effective in biomarker positive adult cancers and in some cases, pediatric patients whose tumors lacked the appropriate biomarker were enrolled. One example of delayed development in pediatric malignancies is the BRAF 
inhibitor vemurafenib. In 2011, vemurafenib was approved by the FDA for unresectable or metastatic melanoma harboring BRAF V600E mutations [47]. In 2010 , it was reported that a proportion of pediatric gliomas harbored the BRAF V600E mutation, yet the first clinical trial of vemurafenib in pediatric patients with recurrent or refractory BRAF-mutant gliomas did not open until 2014 [48]. Of note, a phase 1 trial of the BRAF V600E inhibitor dabrafenib and a phase $1 / 2$ of the MEK inhibitor selumetinib were initiated prior to this. An example of a phase 2 trial of a targeted therapy enrolling biomarker positive and biomarker unknown pediatric patients is the phase $1 / 2$ trial of an ALK inhibitor crizotinib in pediatric patients with refractory solid tumors or anaplastic large-cell lymphoma [49]. The trial had a three-part design: a dose escalation component for all patients (with or without confirmed ALK biomarker) to determine the maximum tolerated dose, an expanded cohort of patients with confirmed ALK variants, and an additional cohort of neuroblastoma patients. The results of this phase $1 / 2$ trial highlight both the promise and challenges in bringing targeted therapies to pediatric malignancies using this type of trial design. Dramatic and durable responses were observed in anaplastic large-cell lymphoma and inflammatory myofibroblasic tumors harboring ALK fusions. There were mixed results in neuroblastoma patients because only a subset of the enrolled neuroblastoma patients had ALK variants and the ALK mutations present in neuroblastoma are different than the ALK variants in lung cancer, the disease for which crizotinib was developed.

More recently, there has been an effort to initiate early phase trials of targeted therapies in pediatric patients earlier in the clinical development pipeline. A particularly successful example is the phase 1 trial of the Trk inhibitor larotrectinib for TRK fusion positive malignancies. Preliminary results from larotrectinib phase 1 trials in pediatric and adult patients, which were recently presented together, showed an objective RR of 91\%. There were no responses in patients whose tumors lacked TRK fusions [50"].

\section{Disease-specific precision trials in newly diagnosed patients}

The ultimate goal of precision medicine is to increase cure rates and decrease toxicity of cancer treatment. This will only be realized when we have incorporated, into standard care of newly diagnosed patients, clinical sequencing for more refined risk stratification and/or, as an indication to utilize matched targeted therapy in upfront treatment regimens. As with umbrella trials, precision trials in newly diagnosed patients require an understanding of the gene variants likely to be encountered in a specific diagnosis. In addition, there should be disease-specific evidence linking gene variants to prognosis and/or activity of targeted therapies. Several trials are profiling leukemia samples from patients newly diagnosed ALL for the presence of the Ph-like expression signature and/or for the presence of $\mathrm{Ph}$ like ALL associated variants. These trials are evaluating outcomes for patients who receive standard ALL therapy in combination with tyrosine kinase inhibitors for BCR-ABL positive or Philadelphia-like leukemia with kinase variants and JAK inhibitors for leukemias with JAK/STAT alterations (Table 3).

In medulloblastoma, large-scale sequencing efforts have revealed four distinct molecular subgroups with significant implications for prognosis and treatment [51,52"]. Clinical trials in newly diagnosed medulloblastoma patients are now incorporating upfront molecular sequencing for risk stratification and treatment assignment. St. Jude's current study for newly diagnosed medulloblastoma patients is evaluating whether good outcomes seen in the WNT molecular subgroup are maintained with reduced treatment intensity. For patients with sonic hedgehog (SHH) pathway mutated medulloblastoma, a SHH inhibitor is being added as a maintenance therapy after conventional cytotoxic chemotherapy to determine whether this strategy improves outcomes for this subset.

One unifying feature in precision trials in newly diagnosed patients with pediatric malignancies is worth noting. Because of the small size of the genomically defined subgroup selected for intervention, all utilize a single-arm design as opposed to randomized design. In other words, the impact of the intervention in the genomically defined patient population will be assessed via comparison with a historical population. The inability to conduct randomized trials is likely to be a common issue in precision trials for newly diagnosed pediatric oncology patients and strategies to address potential challenges encountered when analyzing results of single-arm trials are likely to be needed.

\section{CONCLUSION AND FUTURE DIRECTIONS}

The field of precision medicine in pediatric oncology has seen a number of advances in the last decade, and there are now a range of precision trials available for children with cancer. The initial set of pediatric precision oncology trials will improve our understanding of the clinical impact of targeted therapies. Correlative biology studies embedded in these precision trials (such as efforts to use cell free DNA assays for detecting gene variants) and sharing of resulting clinical and genomic data elements for secondary 
analyses will lead to further understanding of the pediatric cancer genome and new discoveries. Finally, continued drug development, discovery sequencing and preclinical biologic investigation are likely to expand opportunities for precision oncology in pediatric cancers.

\section{Acknowledgements}

None.

\section{Financial support and sponsorship}

None.

\section{Conflicts of interest}

There are no conflicts of interest.

\section{REFERENCES AND RECOMMENDED \\ READING}

Papers of particular interest, published within the annual period of review, have been highlighted as:

- of special interest

- of outstanding interest

1. Siegel RL, Miller KD, Jemal A. Cancer statistics. CA Cancer J Clin 2016; 66:7-30.

2. Armstrong GT, Chen $\mathrm{Y}$, Yasui $\mathrm{Y}$, et al. Reduction in late mortality among 5-year survivors of childhood cancer. N Engl J Med 2016; 374:833-842.

3. National Cancer Institute childhood cancer genomics gaps and opportunities: identification of research priorities workshop summary. 2015:1-33.

4. Loh ML, Zhang J, Harvey RC, et al. Tyrosine kinome sequencing of pediatric acute lymphoblastic leukemia: a report from the Children's Oncology Group TARGET Project. Blood 2013; 121:485-488.

5. Gadd S, Huff V, Walz AL, et al. A Children's Oncology Group and TARGET initiative exploring the genetic landscape of Wilms tumor. Nat Genet 2017; 49:1487-1494.

6. Chun HJ, Lim EL, Heravi-Moussavi A, et al. Genome-wide profiles of extracranial malignant rhabdoid tumors reveal heterogeneity and dysregulated developmental pathways. Cancer Cell 2016; 29:394-406.

7. Pugh TJ, Morozova O, Attiyeh EF, et al. The genetic landscape of high-risk neuroblastoma. Nat Genet 2013; 45:279-284.

8. Downing JR, Wilson RK, Zhang J, et al. The pediatric cancer genome project. Nat Genet 2012; 44:619-622.

9. Lawrence MS, Stojanov $\mathrm{P}$, Mermel $\mathrm{CH}$, et al. Discovery and saturation analysis of cancer genes across 21 tumour types. Nature 2014; 505:495-501.

10. Vogelstein B, Papadopoulos N, Velculescu VE, et al. Cancer genome landscapes. Science 2013; 339:1546-1558.

11. Mody RJ, Wu YM, Lonigro RJ, et al. Integrative clinical sequencing in the management of refractory or relapsed cancer in youth. JAMA 2015; 314:913-925.

12. Harris MH, DuBois SG, Glade Bender JL, et al. Multicenter feasibility study of tumor molecular profiling to inform therapeutic decisions in advanced pediatric solid tumors: the Individualized Cancer Therapy (iCat) study. JAMA Oncol $2016 ; 2: 608-615$

13. Oberg JA, Glade Bender JL, Sulis ML, et al. Implementation of next generation sequencing into pediatric hematology-oncology practice: moving beyond actionable alterations. Genome Med 2016; 8:133.

14. Parsons DW, Roy A, Yang $Y$, et al. Diagnostic yield of clinical tumor and germline whole-exome sequencing for children with solid tumors. JAMA Oncol 2016; 2:616-624.

15. Worst $\mathrm{BC}$, van Tilburg CM, Balasubramanian GP, et al. Next-generation personalised medicine for high-risk paediatric cancer patients - the INFORM pilot study. Eur J Cancer 2016; 65:91-101.

16. Harttrampf AC, Lacroix L, Deloger M, et al. MOlecular Screening for CAncer Treatment Optimization (MOSCATO-01) in pediatric patients: a single institutional prospective molecular stratification trial. Clin Cancer Res 2017; 23:6101-6112.

17. Pincez T, Clement N, Lapouble $\mathrm{E}$, et al. Feasibility and clinical integration of molecular profiling for target identification in pediatric solid tumors. Pediatr Blood Cancer 2017; 64:e26365

18. Mody RJ, Prensner JR, Everett J, et al. Precision medicine in pediatric

- oncology: lessons learned and next steps. Pediatr Blood Cancer 2017; 64:e26288.

This is a review of the initial clinical sequencing studies in pediatric oncology.
19. Seibel NL, Janeway $\mathrm{K}$, Allen $\mathrm{CE}$, et al. Pediatric oncology enters an era of 1. precision medicine. Curr Probl Cancer 2017; 41:194-200.

The review discusses the pediatric Molecular Analysis for Therapy Choice (MATCH) trial in detail.

20. Tran TH, Shah AT, Loh ML. Precision medicine in pediatric oncology: translating genomic discoveries into optimized therapies. Clin Cancer Res 2017; 23:5329-5338.

21. Tasian SK, Hunger SP. Genomic characterization of paediatric acute lym-

- phoblastic leukaemia: an opportunity for precision medicine therapeutics. Br J Haematol 2017; 176:867-882.

This is a review of the genomic alterations seen in acute lymphoblastic leukemia forming the basis of precision trials in newly diagnosed patients.

22. Janeway KA. Molecular profiling in the clinic: moving from feasibility assessment to evaluating clinical impact. Pediatr Blood Cancer 2017; 64:e26482.

23. Janeway KA, Place $A E$, Kieran $M W$, Harris $M H$. Future of clinical genomics in pediatric oncology. J Clin Oncol 2013; 31:1893-1903.

24. Marron JM, DuBois SG, Glade Bender J, et al. Patient/parent perspectives on genomic tumor profiling of pediatric solid tumors: the Individualized Cancer Therapy (iCat) experience. Pediatr Blood Cancer 2016; 63: 1974-1982.

25. Abrams J, Conley B, Mooney M, et al. National Cancer Institute's precision medicine initiatives for the new National Clinical Trials Network. Am Soc Clin Oncol Educ Book 2014; 71-76.

26. Renfro LA, An MW, Mandrekar SJ. Precision oncology: a new era of cancer clinical trials. Cancer Lett 2017; 387:121-126.

27. Allen CE, Laetsch TW, Mody R, et al. Target and agent prioritization for the

- Children's Oncology Group-National Cancer Institute Pediatric MATCH trial. J Natl Cancer Inst 2017; 109:djw274.

This is an in-depth discussion of potentially actionable molecular alterations in childhood malignancies and the process of selecting molecularly targeted therapies for the pediatric MATCH trial.

28. Coyne GO, Takebe N, Chen AP. Defining precision: the precision medicine initiative trials NCl-MPACT and NCl-MATCH. Curr Probl Cancer 2017; 41:182-193.

29. Mullard A. NCl-MATCH trial pushes cancer umbrella trial paradigm. Nat Rev Drug Discov 2015; 14:513-515.

30. McNeil C. NCl-MATCH launch highlights new trial design in precisionmedicine era. J Natl Cancer Inst 2015; 107:djv193.

31. Moreno L, Pearson ADJ, Paoletti X, et al. Early phase clinical trials of antic-

- ancer agents in children and adolescents - an ITCC perspective. Nat Rev Clin Oncol 2017; 14:497-507.

This is a perspective article laying out the framework for the European approach to early phase precision trials.

32. Vassal G, Rousseau R, Blanc $P$, et al. Creating a unique, multistakeholder paediatric oncology platform to improve drug development for children and adolescents with cancer. Eur J Cancer 2015; 51:218-224.

33. Pearson AD, Herold R, Rousseau R, et al. Implementation of mechanism of action biology-driven early drug development for children with cancer. Eur J Cancer 2016; 62:124-131.

34. Gilles Vassal GRCC. Accelerating Pediatric Drug Development Meeting, February, 2017, Friends of Cancer Research, Washington, DC.

35. Den Boer ML, van Slegtenhorst $M$, De Menezes RX, et al. A subtype of childhood acute lymphoblastic leukaemia with poor treatment outcome: a genome-wide classification study. Lancet Oncol 2009; 10:125-134.

36. Roberts KG, Li Y, Payne-Turner D, et al. Targetable kinase-activating lesions in Ph-like acute lymphoblastic leukemia. N Engl J Med 2014; 371: 1005-1015.

37. Wells J, Jain N, Konopleva M. Philadelphia chromosome-like acute lymphoblastic leukemia: progress in a new cancer subtype. Clin Adv Hematol Oncol 2017; 15:554-561.

38. Tasian SK, Teachey DT, Li Y, et al. Potent efficacy of combined PI3K/mTOR and JAK or $A B L$ inhibition in murine xenograft models of Ph-like acute lymphoblastic leukemia. Blood 2017; 129:177-187.

39. Jain $\mathrm{N}$, Roberts $\mathrm{KG}$, Jabbour $\mathrm{E}$, et al. Ph-like acute lymphoblastic leukemia: a high-risk subtype in adults. Blood 2017; 129:572-581.

40. Kim ES, Bruinooge SS, Roberts $S$, et al. Broadening eligibility criteria to make clinical trials more representative: American Society of Clinical Oncology and Friends of Cancer Research Joint Research Statement. J Clin Oncol 2017; 35:3737-3744.

41. Chuk MK, Mulugeta $Y$, Roth-Cline $M$, et al. Enrolling adolescents in disease/ target-appropriate adult oncology clinical trials of investigational agents. Clin Cancer Res 2017; 23:9-12.

42. Schleiermacher G, Javanmardi N, Bernard V, et al. Emergence of new ALK mutations at relapse of neuroblastoma. J Clin Oncol 2014; 32:2727-2734.

43. Padovan-Merhar OM, Raman $P$, Ostrovnaya I, et al. Enrichment of targetable mutations in the relapsed neuroblastoma genome. PLoS Genet 2016; 12:e1006501.

44. Eleveld TF, Oldridge DA, Bernard V, et al. Relapsed neuroblastomas show frequent RAS-MAPK pathway mutations. Nat Genet 2015; 47: 864-871.

45. Schramm A, Koster J, Assenov Y, et al. Mutational dynamics between primary and relapse neuroblastomas. Nat Genet 2015; 47:872-877. 
46. Wood AC, Krytska K, Ryles HT, et al. Dual ALK and CDK4/6 inhibition demonstrates synergy against neuroblastoma. Clin Cancer Res 2017; 23:2856-2868.

47. FDA approves vemurafenib for treatment of metastatic melanoma. Oncology (Williston Park) 2011; 25:906.

48. Dougherty MJ, Santi M, Brose MS, et al. Activating mutations in BRAF characterize a spectrum of pediatric low-grade gliomas. Neuro Oncol 2010; 12:621-630.

49. Mosse YP, Lim MS, Voss SD, et al. Safety and activity of crizotinib for paediatric patients with refractory solid tumours or anaplastic large-cell lymphoma: a Children's Oncology Group phase 1 consortium study. Lancet Oncol 2013; 14:472-480.
50. Laetsch TW, DuBois SG, Nagasubramanian $R$, et al. A pediatric phase I study

- of larotrectinib, a highly selective inhibitor of the tropomyosin receptor kinase (TRK) family. J Clin Oncol 2017; 35(15_suppl):10510.

This is the presentation of a recently completed phase $1 / 2$ trial of a molecularly targeted therapy conducted simultaneously in children and adults.

51. Shih DJ, Northcott PA, Remke $M$, et al. Cytogenetic prognostication within medulloblastoma subgroups. J Clin Oncol 2014; 32:886-896.

52. Northcott PA, Buchhalter I, Morrissy AS, et al. The whole-genome landscape - of medulloblastoma subtypes. Nature 2017; 547:311-317.

The discovery sequencing study updates our understanding of the molecular subtypes of medulloblastoma on which the current precision trials in this disease are based. 